\title{
Compensation for the Effects of Head Acceleration on Jaw Movement in Speech
}

\author{
Douglas M. Shiller, ${ }^{1}$ David J. Ostry, ${ }^{1,2}$ Paul L. Gribble, ${ }^{3}$ and Rafael Laboissière ${ }^{4}$ \\ ${ }^{1}$ Department of Psychology, McGill University, Montreal, Quebec, Canada H3A 1B1, 2Haskins Laboratories, New Haven, \\ Connecticut 06511, 3University of Western Ontario, London, Ontario, Canada N6A 5C2, and 4/nstitute of Speech \\ Communication, 38031 Grenoble, France
}

Recent studies have demonstrated the ability of subjects to adjust the control of limb movements to counteract the effects of self-generated loads. The degree to which subjects change control signals to compensate for these loads is a reflection of the extent to which forces affecting movement are represented in motion planning. Here, we have used empirical and modeling studies to examine whether the nervous system compensates for loads acting on the jaw during speech production. As subjects walk, loads to the jaw vary with the direction and magnitude of head acceleration. We investigated the patterns of jaw motion resulting from these loads both in locomotion alone and when locomotion was combined with speech production. In locomotion alone, jaw movements were shown to vary systematically in direction and magnitude in relation to the acceleration of the head. In contrast, when locomotion was combined with speech, variation in jaw position during both consonant and vowel production was substantially reduced. Overall, we have demonstrated that the magnitude of load associated with head acceleration during locomotion is sufficient to produce a systematic change in the position of the jaw. The absence of variation in jaw position during locomotion with speech is thus consistent with the idea that in speech, the control of jaw motion is adjusted in a predictive manner to offset the effects of head acceleration.

Key words: speech; jaw; locomotion; compensation; dynamics; mathematical model
Speech production occurs in the context of numerous and often complex loads. This includes self-generated loads on vocal tract structures that arise because of the motion of mechanically coupled articulators, analogous to interaction torques in limb movement, as well as forces on orofacial articulators that arise from head and body motions. These loads may interfere with the function of vocal tract structures and accordingly may be compensated for by the nervous system in orofacial motion planning.

In the case of arm movements, several recent studies have explored the ability of subjects to adapt the control of movement to produce seemingly unperturbed movements in the presence of self-generated loads. This ability has been demonstrated both for loads such as artificial force fields (Lackner and DiZio, 1994; Shadmehr and Mussa-Ivaldi, 1994; Gandolfo et al., 1996; Conditt et al., 1997; Goodbody and Wolpert, 1998) and for naturally occurring loads such as joint interaction torques in multijoint movements (Sainburg et al., 1993; Almeida et al., 1995; Cooke and Virji-Babul, 1995; Sainburg et al., 1995; Gribble and Ostry, 1999; Koshland et al., 2000). To achieve this adaptation, the nervous system must presumably take into account limb dynamics and external loads to perform the adjustments that offset the load. It has been suggested that this sort of predictive compensation may be attributed to "internal models" of the motor system.

Although several researchers have suggested that as in the

\footnotetext{
Received Nov. 17, 2000; revised May 31, 2001; accepted May 31, 2001.
}

This research was supported by National Institutes of Health Grant DC-00594 from the National Institute on Deafness and Other Communication Disorders, by Natural Sciences and Engineering Research Council-Canada, and by Fonds pour la Formation de Chercheurs et l'Aide à la Recherche-Quebec.

Correspondence should be addressed to Dr. D. J. Ostry, Department of Psychology, McGill University, 1205 Dr. Penfield Avenue, Montreal, Quebec, Canada H3A 1B1. E-mail: ostry@motion.psych.mcgill.ca.

Copyright (C) 2001 Society for Neuroscience $\quad 0270-6474 / 01 / 216447-10 \$ 15.00 / 0$ limb, control signals for speech are based on a predictive internal representation of the vocal tract (Guenther, 1995; Guenther et al., 1998; Houde and Jordan, 1998; Perkell et al., 1997), evidence to date suggests that adaptation in the orofacial system is much less complete, or less successful, than that observed in the motion of the limbs. For example, in speech, subjects do not completely compensate for loads acting on the tongue and the jaw that arise because of changes in head orientation relative to gravity (Shiller et al., 1999; Tiede et al., 2000). A degree of predictive compensation has been observed in electromyographic (EMG) responses to loads applied during cyclical jaw movements (Abbink et al., 1998, 1999). However, in these studies, anticipatory adjustments to jaw muscle activity were not large in comparison with sensorybased responses and were not observed in all subjects. Limited adaptation is similarly observed when vocal tract geometry is modified via the use of artificial palates (McFarland et al., 1996; Honda and Kaburagi, 2000) and bite blocks (McFarland and Baum, 1995).

In arm movement research, some of the most compelling evidence of predictive compensation comes from work involving naturally occurring, self-generated loads. This includes grip force adjustment during rapid arm movements with handheld loads, preparatory changes in trunk and leg muscles in anticipation of arm movement, and adjustments to arm muscle activity in anticipation of interaction forces that arise because of multijoint dynamics (Horak et al., 1984; Flanagan et al., 1993; Flanagan and Wing, 1997; De Wolf et al., 1998; Gribble and Ostry, 1999). In the present paper, we describe an analog of these manipulations in the orofacial system. Specifically, we have used a combination of empirical and modeling studies to examine the extent to which subjects compensate for loads on the jaw in speech that arise during locomotion. 


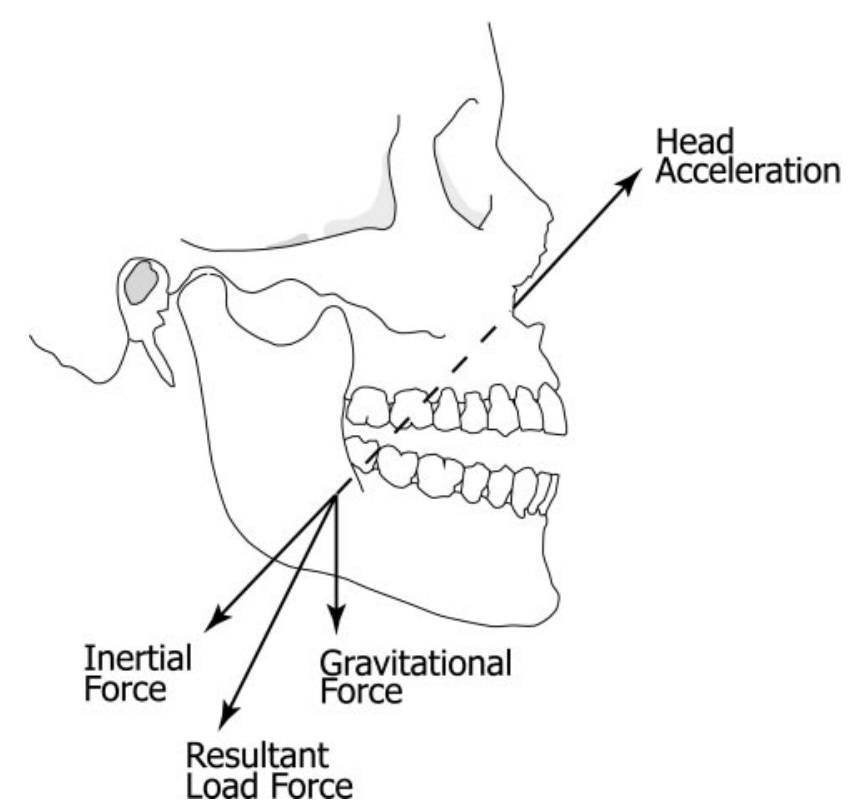

Figure 1. As the head accelerates during locomotion, a force is applied to the jaw that is proportional in magnitude but acts in the opposite direction.

As subjects walk or run, forces act on the jaw that vary with the acceleration of the head (Fig. 1). Upward acceleration produces a downward load on the jaw, and downward acceleration produces an upward load. By examining jaw kinematic patterns associated with loads caused by head acceleration, we can address the extent to which the control of speech movements is modified to offset the effects of these loads. If control signals are adjusted, then upward and downward head acceleration during locomotion, which can be shown to be sufficient to affect jaw movement, may have little effect on the position of the jaw during speech production.

\section{MATERIALS AND METHODS}

Empirical study. Eight subjects were tested, with none reporting any history of speech motor disorder or temperomandibular joint dysfunction. Subjects repeated a series of speech sequences while either walking or running on a treadmill. Each sequence consisted of a consonantvowel-consonant (CVC) combination embedded in a carrier sentence. Four CVC sequences were tested, resulting from the combination of the consonants $s$ and $k$ with the vowel sounds $a$ (as in "cat") and $e$ (as in "fed"). The vowels were chosen to vary the amplitude of jaw movement; the consonants were chosen to vary the jaw position at closure. The test sentences were of the form "see sasy again" or "see keky again." By using the same speech sound immediately preceding and following the CVC, context-dependent variation in movement was minimized.

A motor-driven treadmill (Precor, Bothell, WA) was used to manipulate the speed of locomotion and hence the magnitude of head acceleration. Three speeds were chosen to vary the magnitude of head acceleration: $2 \mathrm{mph}$ (slow walking), $4 \mathrm{mph}$ (fast walking), and $6 \mathrm{mph}$ (jogging).

Subjects repeated the test sentence at a self-chosen rate while walking or running at each of the three locomotion speeds. In all, 12 conditions were tested (three locomotion speeds $\times$ four CVC sequences) in a series of 5 min blocks. Each subject was tested in a unique, fully randomized block order. Because the production of the test utterances was self-paced, the total number of repetitions was not identical across locomotion speeds. Subjects tended to produce fewer repetitions at the $6 \mathrm{mph}$ condition; however enough time was provided to produce a large number of repetitions in each of the 12 experimental conditions $(\sim 100)$.

A further condition was tested in which subjects walked or ran on the treadmill without speaking. For this task, subjects were instructed not to clench their teeth but were given no further instructions with regard to the position of their jaw. Data were collected during two 5 min blocks at each of the three locomotion speeds. The order of testing was fully randomized.

Motion of the head and jaw was recorded using Optotrak (Northern Digital, Waterloo, Ontario, Canada), an optoelectronic position measurement system that tracks the three-dimensional motion of infraredemitting diodes (IREDs). To track head motion, four IREDs were attached to an acrylic and metal dental appliance (weight, $10 \mathrm{gm}$ ) that was custom-made for each subject and fixed with a dental adhesive (Iso-Dent; Ellman International, Hewlett, NY) to the buccal surface of the maxillary teeth. Similarly, to track motion of the jaw, four IREDs were attached to an appliance affixed to the mandibular teeth. In both cases, the four IREDs were arranged in a rectangular configuration in the frontal plane. The dental appliances had little effect on the intelligibility of the utterances tested in this study. IRED motion was measured at a sampling rate of $200 \mathrm{~Hz}$.

To explore fully the effect of head acceleration on jaw movement during speech, it was necessary that subjects produce large upward and downward head accelerations coincident with production of each of the initial consonant, vowel, and final consonant portions of the CVC. Subjects were therefore encouraged not to synchronize their speech with the locomotion cycle to vary the timing of sentence repetition with respect to locomotion. Compliance with this instruction was monitored on-line by the experimenter and later verified by examining the recorded jaw and head movement traces (see Data analysis).

Although the interval between repetitions of the test sentence was allowed to vary, subjects were instructed to maintain a constant speech volume and speech rate. Speech acoustics were monitored on-line by the experimenter, with feedback given to the subject if speech rate or volume varied significantly. The speech acoustical signal was also recorded digitally using a small microphone (Audio-Technica, Stow, $\mathrm{OH}$ ) taped to the bridge of the nose. Speech rate and volume were examined quantitatively on the basis of the recorded acoustic signal (see Data analysis). A more detailed acoustical analysis was not possible because of treadmill noise and the large acoustical artifact produced by footfall.

Data analysis. The three-dimensional position data for each IRED were digitally low-pass filtered using a second-order zero-phase lag Butterworth filter with a cutoff frequency of $10 \mathrm{~Hz}$ (chosen on the basis of Fourier analysis and then verified by comparison of raw and filtered data). The original camera-centered representation of jaw motion was transformed into a six-dimensional rigid-body representation of jaw position and orientation in a head-centered coordinate frame [for details, see Ostry et al. (1997)]. The origin of this new coordinate system is the condyle center (projected onto the midsagittal plane) when the jaw is at occlusion. The "horizontal" axis is aligned with the occlusal plane. The estimated orientation of the occlusal plane is accurate to within approximately $\pm 1^{\circ}$. Inaccuracies are reflected as constant offsets within the data. Data analysis of jaw motion focused on sagittal plane rotation; this constitutes the largest source of jaw motion in speech production (Ostry and Munhall, 1994; Ostry et al., 1997).

An examination of head motion revealed large magnitudes of vertical head acceleration and relatively small magnitudes of acceleration in the horizontal plane. The average maximum vertical acceleration across subjects was approximately $\pm 13 \mathrm{~m} / \mathrm{sec}^{2}$, whereas the maximum horizontal acceleration was approximately $\pm 2 \mathrm{~m} / \mathrm{sec}^{2}$. Accordingly, the analyses reported below focus on vertical head acceleration and the corresponding jaw orientation in the sagittal plane.

An interactive computer program was used to extract the kinematic data associated with the CVC portion of the utterance from the surrounding carrier sentence. The kinematic and acoustical data were displayed simultaneously. The start and end of each CVC were initially identified on the basis of the acoustical signal. In the kinematic record, CVC production is typically associated with a large-amplitude movement of the jaw corresponding in time to the voiced portion of the CVC utterance. Zero-crossings in jaw velocity were used to obtain positions and times for initial consonant production (movement start), vowel production (position of maximum opening), and production of the final consonant (movement end). The positions of selected zero-crossings were overlaid on the kinematic trace for verification. For cases in which the position of the zero-crossing clearly failed to coincide with the production of the consonant or vowel (on the basis of visual inspection), the data were discarded for that particular movement. In particular, data were rejected when zero-crossings, corresponding to consonant positions, did not appear to coincide with the start or end of the large-amplitude jaw opening and closing movement associated with the CVC, judging 

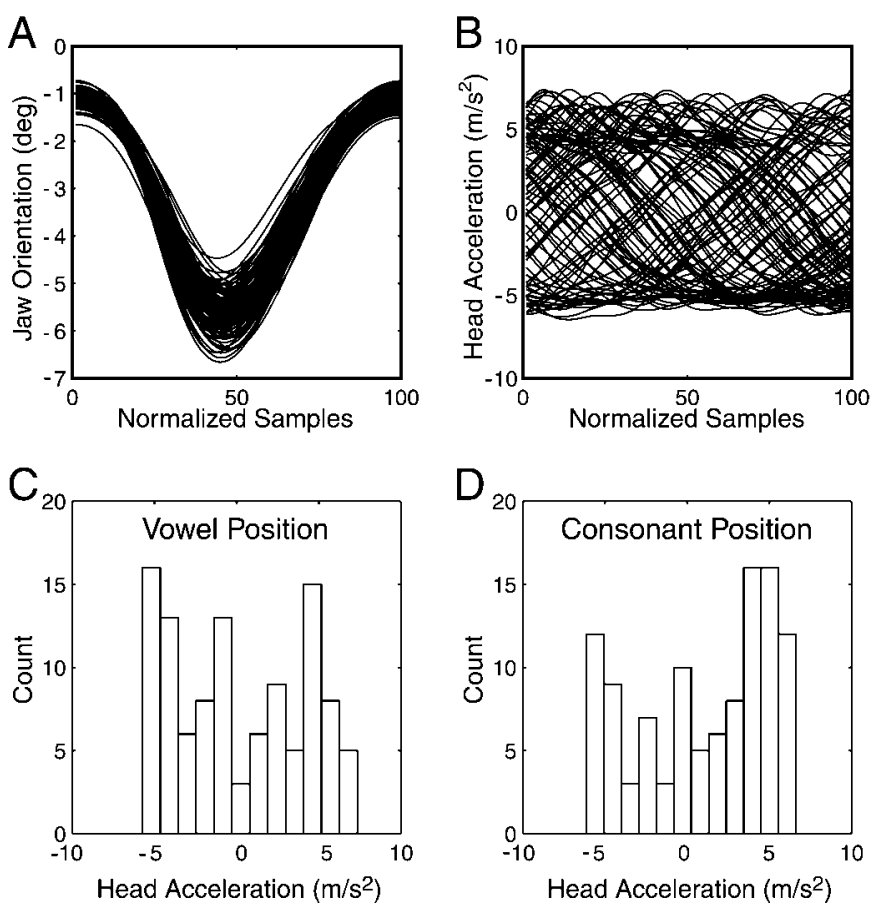

Figure 2. Subjects were successful in producing a range of vertical head accelerations at the consonant and vowel positions of the CVC utterance. This figure shows data from a single subject at the $4 \mathrm{mph}$ locomotion speed for the utterance "sas." $A$, All jaw movements for the production of this CVC, isolated from the carrier sentence and time normalized to 100 samples. $B$, Head vertical acceleration coinciding in time with each of the jaw movements in $A$. It can be seen that there is no systematic relationship between the timing of jaw movement and head acceleration. $C, D$, Histograms of head acceleration coinciding with production of the vowel $(C)$ and the first consonant $(D)$. Positive accelerations are in the upward direction. In both cases a broad range of acceleration magnitudes and directions are observed. deg, Degree.

from the timing of the movement peak and the audio signal. Two to three percent of observations were omitted for this reason (see Discussion).

As noted above, subjects were instructed to avoid synchronizing their speech with the locomotion cycle so that production of the CVC would coincide with a range of upward and downward head accelerations. The distribution of head accelerations at the time of consonant and vowel production was examined for all subjects. This revealed a wide range of positive (upward) and negative (downward) accelerations, indicating that subjects were successful in following this instruction (Fig. 2).

The data were selected for analysis as follows. Values for jaw orientation and head vertical acceleration were obtained at the time of the first consonant, the vowel, and the second consonant. This was done separately for each repetition of the test utterance. In each of the four CVC conditions and three locomotion speeds, we selected for further analysis the observations associated with the 10 largest upward and 10 largest downward head accelerations. Recall that the overall goal of this study is to determine whether subjects compensate for the forces acting on the jaw that arise because of head acceleration (that is, whether jaw positions are maintained in spite of differences in the direction and magnitude of load). Thus, by examining the effects of only the largest head accelerations, we increase the likelihood of observing a difference in jaw position, which would constitute evidence against compensation.

In trials involving locomotion without speech, the data were selected on the basis of head acceleration. Successive maxima and minima corresponding to maximum upward and maximum downward head acceleration were identified. These values and the corresponding jaw orientations formed the data set for each locomotion speed. As in the case of locomotion with speech, at each locomotion speed, the observations associated with the 10 largest upward and 10 largest downward head accelerations were selected for further analysis.

A normalization procedure was performed to examine jaw orientation data across subjects. In the speech condition, the data were normalized

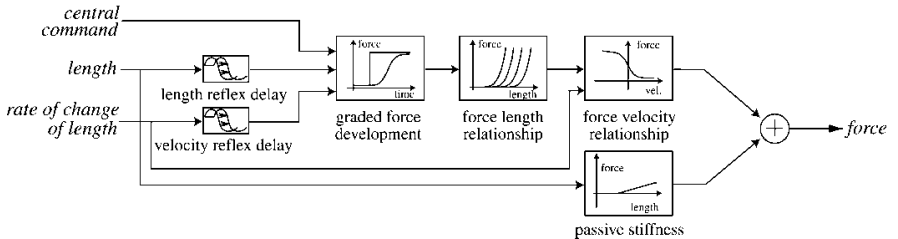

Figure 3. Schematic of the muscle model used in the simulation studies. vel., Velocity.

separately for each initial consonant, vowel, and final consonant. The normalization involved calculating deviation scores by subtracting the mean separately for each of the experimental conditions (three locomotion speeds $\times$ four $\mathrm{CVC}$ conditions). In the nonspeech condition, the jaw orientation data for each locomotion speed were converted to deviation scores using the mean of each condition, again on a per-subject basis. The procedure preserves differences in jaw orientation that arise because of direction and magnitude of head acceleration but expresses the effects of head acceleration about a transformed mean of zero. The procedure removes variability caused by factors such as the position of the jaw during production of the test utterances. For example, the jaw is in a lower overall position for the production of $k$ than for the production of $s$. Moreover, individual subjects produce these same speech sounds with different jaw positions. The normalization corrects for these factors and permits comparisons across conditions and subjects.

Simulation studies. Computer simulations were performed to generate predicted patterns of jaw motion on the assumption that subjects make no adjustment to control signals to compensate for loads arising because of acceleration of the head. If the predicted patterns of jaw movement are comparable with those observed empirically, an absence of compensation would be indicated.

The simulation studies use a recently developed model of sagittal plane jaw and hyoid movement (Laboissiere et al., 1996). The model includes seven muscle groups corresponding to the masseter/medial pterygoid, anterior temporalis, posterior temporalis, lateral pterygoid, anterior digastric, posterior digastric, and sternohyoid [for schematic, see Laboissiere et al. (1996)]. These muscles contribute to motion in four kinematic degrees of freedom: the horizontal jaw position, sagittal plane jaw orientation (about an axis through the center of the mandibular condyle), hyoid bone horizontal position, and hyoid vertical position. The model includes neural control signals, position- and velocity-dependent reflexes, and muscle mechanical properties. It also includes jaw and hyoid bone dynamics (including forces acting on the jaw because of head motion) and realistic musculoskeletal geometry. The muscle mechanical model is a variant of the standard Hill model [for review, see Zajac (1989)] and includes force generation due to the contractile element (the dependence of force on muscle length and velocity), activation dynamics due to calcium kinetics, and the passive dependence of force on muscle length (Fig. 3). Modeled muscle geometry and anthropometrics are taken from standard anatomical sources (Scheideman et al., 1980; McDevitt, 1989). The reader is referred to Ostry et al. (1996) and Shiller et al. (1999) for other studies in which the jaw model is used in conjunction with experimental results.

The modeled control signals are based on the $\lambda$ version of the equilibrium point (EP) hypothesis. According to this hypothesis, shifts in the equilibrium state of the motor system are used to produce movement. The shifts arise from a centrally specified change in $\lambda$, the muscle length at which motoneuron recruitment begins. Muscle activation and hence muscle force vary in proportion to the difference between the actual and threshold muscle length. Motion of the jaw to a new equilibrium position can be achieved via the coordination of $\lambda$ values associated with individual muscles [for a detailed description of the EP formulation, see Feldman et al. (1990)].

In the present model, shifts in values of $\lambda$ for multiple muscles are coordinated to produce motion of the jaw independently in each of its four kinematic degrees of freedom [for details, see Laboissiere et al. (1996)]. These coordinated commands to jaw muscles are analogous to the "R" command described in previous formulations of the model (Feldman, 1986; Feldman et al., 1990).

The ability to coactivate antagonist muscles independent of movement has been documented in both behavioral and physiological studies (Humphrey and Reed, 1983; Milner and Cloutier, 1993; Gribble and Ostry, 1998). Accordingly, in addition to the motor command used to produce 


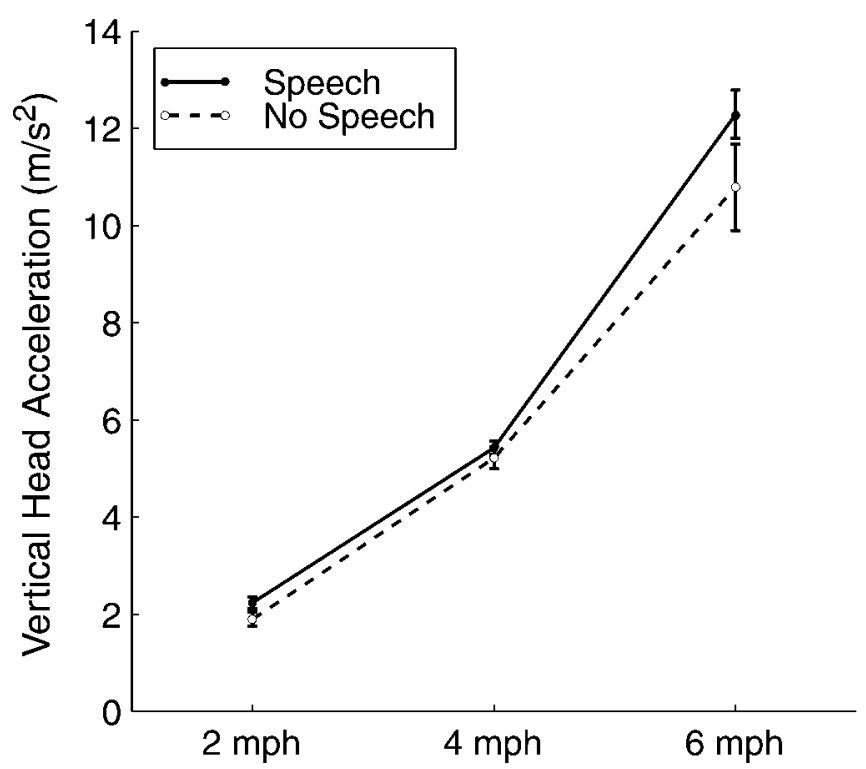

Figure 4. Mean vertical head acceleration ( $\pm 1 \mathrm{SE})$ at each of the three locomotion speeds for locomotion with speech (solid line) and locomotion alone (dashed line). The difference between speech and nonspeech conditions is not statistically significant at any locomotion speed.

movement, the model makes use of an independent set of $\lambda$ shifts that specify the level of muscle coactivation (and hence jaw stiffness) without producing motion. This cocontraction command is analogous to the "C" command described in previous formulations of the model.

To produce speech-like jaw-opening and -closing movements, the simulations presented in this study use coordinated commands for jaw rotation and horizontal translation. A constant rate shift in the equilibrium orientation and equilibrium jaw position is assumed. Consistent with the empirical finding that jaw rotation and translation are time synchronized during speech (Ostry and Munhall, 1994), the specified jaw rotation and translation commands begin and end simultaneously in each opening and closing phase of jaw motion. A constant cocontraction command was used for all simulated movements. (The effects of different cocontraction levels on simulation results are presented in Results.)

\section{RESULTS}

\section{Empirical}

Head acceleration was manipulated by varying the speed of treadmill locomotion (2, 4, and $6 \mathrm{mph}$ ). It was verified that significant differences in head acceleration across locomotion speeds were produced in both the speech and nonspeech conditions. Figure 4 shows mean head acceleration averaged over subjects. Tukey tests were used to verify that for both speech and nonspeech conditions, all pairwise differences were significant ( $p<0.01$ in all cases $)$.

Data from a single subject are shown in Figure 5. Figure $5 A$ shows vertical head acceleration (bottom trace) and the corresponding jaw orientation (top trace) for locomotion without speech (6 mph condition). Jaw orientation is shown relative to the angle at occlusion, with the negative direction corresponding to jaw opening. Head acceleration results in forces to the jaw that act in the opposite direction. Here, the effects can be clearly seen as a phasic change in jaw orientation related to the timing and magnitude of head acceleration. Downward head accelerations (negative) are associated with higher jaw positions, and upward head accelerations (positive) are associated with lower jaw positions. Figure $5 B$ shows the pattern of jaw orientation associated with the repeated utterance "see sasy again" while the head is stationary, that is, in the absence of locomotion. The large-
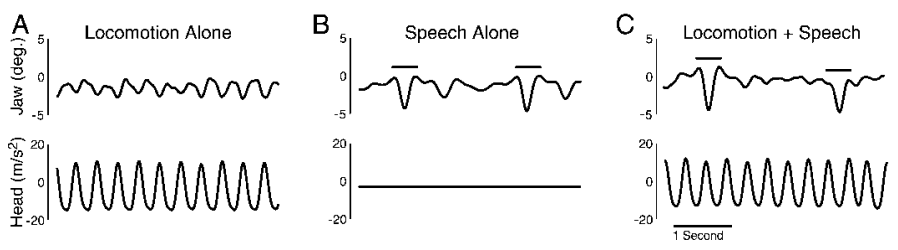

Figure 5. A, Vertical head acceleration (bottom trace) and the corresponding jaw orientation (top trace) for locomotion without speech (6 mph condition). Positive head accelerations are in the upward direction. Jaw opening is in the negative direction. The effect of head acceleration on the jaw can be seen as a phasic change in jaw orientation related to the timing and magnitude of head acceleration. $B$, The pattern of jaw orientation associated with the repeated utterance "see sasy again" while the head is stationary. $C$, Jaw orientation and head acceleration for speech and locomotion combined. In $B$ and $C$, the production of the $C \mathrm{VC}$ is indicated with a horizontal line above the jaw movement trace.
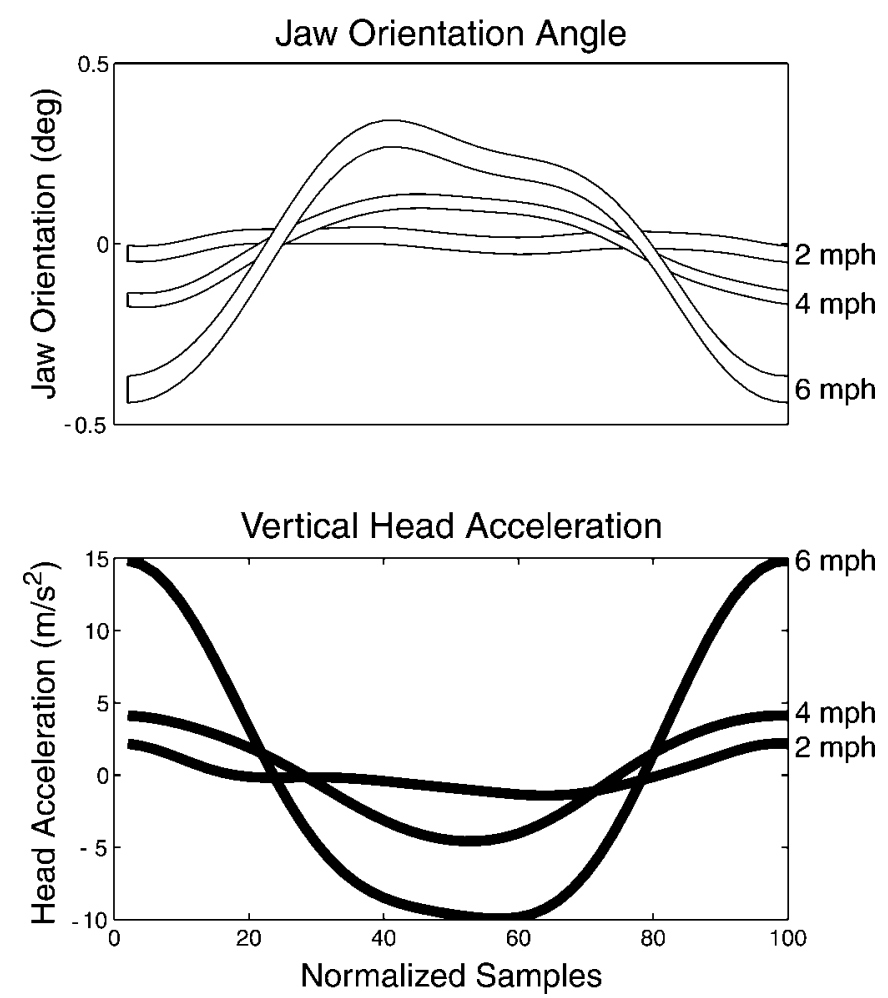

Figure 6. The pattern of jaw motion related to vertical head acceleration during locomotion without speech. Bottom, Mean vertical head acceleration (upward accelerations are positive). Top, The corresponding mean jaw orientation (the downward direction on the figure corresponds to jaw opening). The width of the trace indicates \pm 1 SE (for head acceleration, the lines are thicker for visualization purposes because the magnitude of SE was extremely small).

amplitude opening and closing movement of the jaw, indicated by a horizontal line, is associated with the CVC sequence sas. Figure $5 C$ shows jaw orientation and head acceleration when speech and locomotion are combined.

An example of the relationship between jaw motion and vertical head acceleration is shown for a single subject in Figure 6 . The figure shows the pattern for locomotion in the absence of speech. The plots were generated as follows. The raw data consisted of multiple cycles of head acceleration and associated jaw movement. Successive maxima in upward head acceleration (positive direction) were identified, and individual cycles of head acceleration were extracted. The jaw orientation traces corre- 


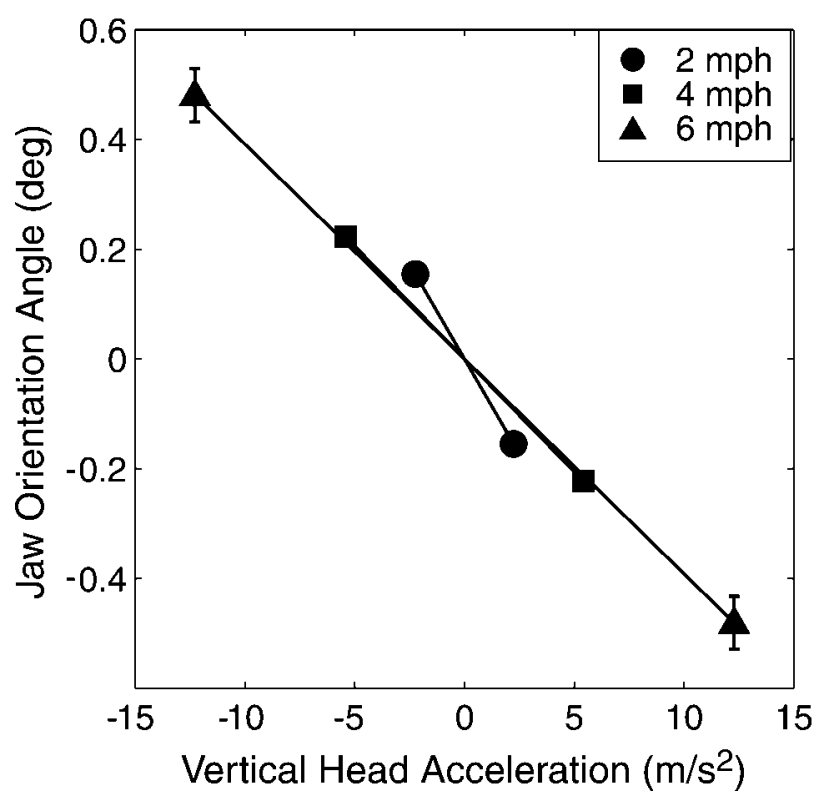

Figure 7. Results averaged over subjects for locomotion without speech. Mean head acceleration is plotted on the $x$-axis (upward acceleration is positive), and the corresponding mean jaw orientation ( $\pm 1 \mathrm{SE})$ is shown on the $y$-axis (the downward direction is jaw opening). The three lines show the jaw orientations associated with three magnitudes of head acceleration. It can be seen that differences in jaw orientation at each locomotion speed vary directly with the magnitude of head acceleration. Note that in the 2 and $4 \mathrm{mph}$ conditions, SEs are less than the size of the plotted symbols. Also note that estimates of variability in head acceleration at each locomotion speed are shown in Figure 4.

sponding in time to cycles of head acceleration were also selected. For each of the three locomotion speed conditions, the jaw and head acceleration data for each cycle were linearly time normalized to 100 samples and then averaged across cycles at each point in time. The bottom panel of Figure 6 shows mean vertical head acceleration, and the top panel shows the corresponding mean jaw orientation. A temporal coupling between head and jaw motion can be seen, with changes in jaw orientation related to the magnitude of vertical head acceleration. It should be noted that the time-normalized records of Figure 6 show the cycle durations as equal to highlight the increase in the amplitude of jaw movement across locomotion speeds. In fact, cycle durations differ with locomotion speed.

Figure 7 shows mean values across subjects for locomotion in the absence of speech. Head acceleration is shown on the $x$-axis, with positive values corresponding to acceleration in the upward direction. Jaw orientation is plotted on the $y$-axis. The orientation values are presented on an axis centered about the overall mean, such that the positive direction is toward occlusion (higher jaw elevation) and the negative direction is away from occlusion (lower jaw elevation). The three lines of Figure 7 show mean values corresponding to the 2, 4, and $6 \mathrm{mph}$ locomotion speeds. The figure shows that at all three speeds, upward head acceleration is associated with a lower jaw position, whereas downward head acceleration is associated with a higher jaw position. Moreover, jaw orientation varies with the magnitude of head acceleration. It should be noted that the lines connecting values of jaw orientation at each locomotion speed are provided as an aid to visualization. Jaw orientations associated with intermediate values of head acceleration were not examined.

Statistical analyses were performed by use of a repeated mea-

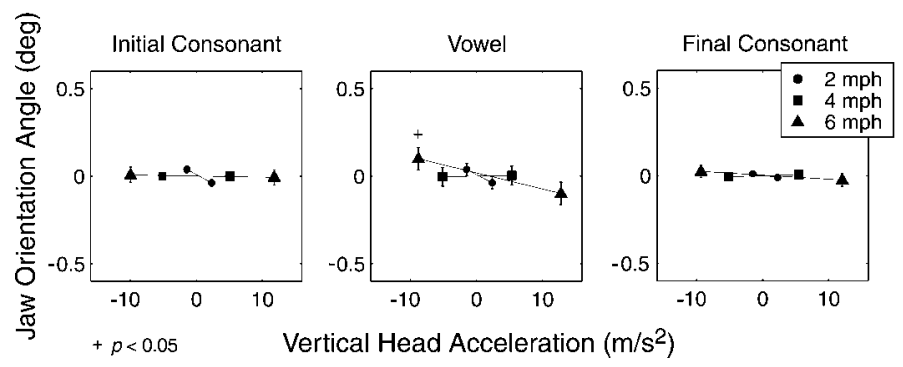

Figure 8. Results averaged over subjects for locomotion with speech. Left, Right, Mean jaw displacement ( $\pm 1 \mathrm{SE})$ associated with three levels of head acceleration during production of the initial (left) and final (right) consonants. Positive head accelerations are in the upward direction; jaw opening is downward. Both panels show that head acceleration has little effect on jaw orientation at any of the three locomotion speeds. Middle, The effect of head acceleration on the jaw during production of the vowel. Here, again, there is little effect in the 2 and $4 \mathrm{mph}$ conditions and a small but significant effect at the $6 \mathrm{mph}$ speed (see Results). Note that in some cases SEs are less than the size of the plotted symbols.

sures ANOVA and post hoc comparisons of pairwise means using Tukey's method. Jaw orientation varied significantly with the direction of head acceleration. Overall, the jaw was lower during upward acceleration than during downward acceleration $(p<$ $0.01)$. Differences in jaw orientation between upward and downward acceleration were similarly reliable at each of the three locomotion speeds $(p<0.01)$. Finally, we examined whether the size of the effect, that is, the difference in jaw orientation between upward and downward head acceleration, differed significantly between the three locomotion speeds. Significant differences were found between the 2 and $6 \mathrm{mph}$ conditions $(p<0.01)$ and between the 4 and $6 \mathrm{mph}$ conditions $(p<0.01)$, but no difference was found between the 2 and $4 \mathrm{mph}$ conditions.

Mean values across subjects for the experimental condition involving locomotion during speech production are shown in Figure 8. Data are averaged across all four CVC conditions and are presented separately for the vowel and two consonant positions associated with production of the CVC. Each panel shows mean jaw orientations at the three locomotion speeds. As in Figure 7, the three lines correspond to the effects of locomotion on the jaw at 2, 4, and $6 \mathrm{mph}$. It can be seen that, in all but one case, the direction and magnitude of head acceleration have little effect on jaw orientation. In the case of vowel production (Fig. 8, middle) at the $6 \mathrm{mph}$ condition, the effect of head acceleration on jaw orientation is somewhat larger.

ANOVA was performed separately to assess the effects of head acceleration on jaw orientation for each of the two consonants and the vowel. In these analyses, no differences were found in jaw orientation between upward and downward head acceleration at either consonant position. In the case of vowel production, a significant difference in jaw orientation was observed only in the 6 mph condition $(p<0.05)$.

Figure 9 shows results for locomotion with speech for each of the four phonetic conditions separately. Each CVC is shown in a separate column. In general, the magnitude of the effect of head acceleration on jaw orientation is small. However, when the data were examined in detail, a number of statistically reliable differences were observed on the basis of post hoc tests. Specifically, during production of the initial and final consonants, differences were found for the CVC conditions sas and sos. Pairwise comparisons of means for the initial and final consonant indicated significant differences in jaw orientation at the $6 \mathrm{mph}$ condition, 

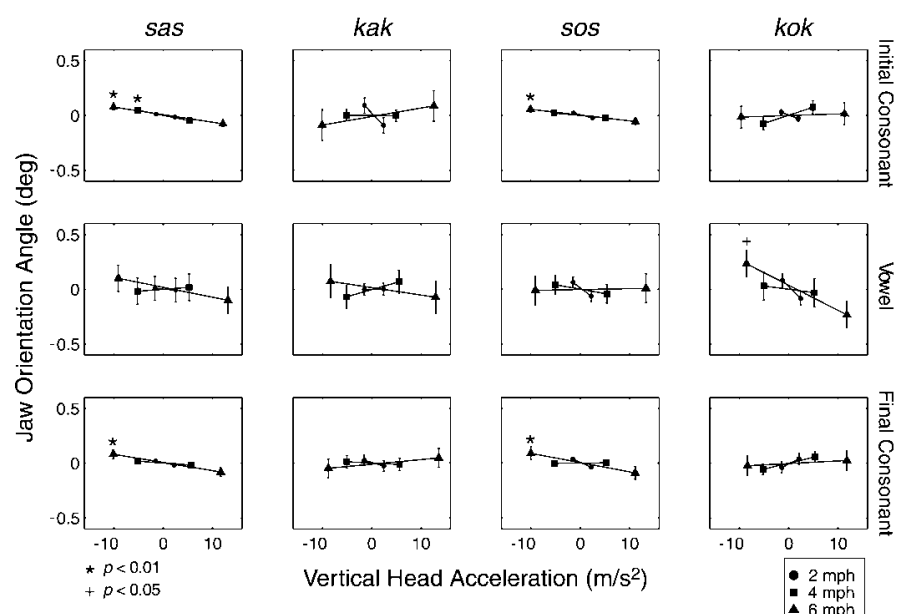

Vertical Head Acceleration $\left(\mathrm{m} / \mathrm{s}^{2}\right)$

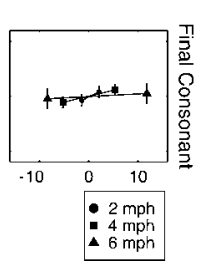

Figure 9. Average results for locomotion with speech, shown for each of the four phonetic conditions separately. Data from each CVC condition are shown in a separate column. Each panel shows mean jaw displacement $( \pm 1 \mathrm{SE})$ associated with three levels of head acceleration during production of either the initial consonant (top tow), vowel (middle row), or final consonant (bottom row). In general, the magnitude of the effect of head acceleration on jaw orientation is small. Significant effects are indicated. SEs are less than the size of the plotted symbols in some cases.

as well as in the $4 \mathrm{mph}$ condition for production of the initial consonant in sas $(p<0.01)$. In the case of vowel production, a single statistically significant difference was observed [for the phonetic condition kok at the $6 \mathrm{mph}$ locomotion speed $(p<$ $0.05)]$. Note that although statistically reliable, these differences are small.

The possibility exists that observed differences in jaw orientation between speech and nonspeech conditions may be caused by differences in the magnitude of head acceleration. We tested for this by examining head accelerations directly. Specifically, an examination of the mean maximum upward and downward head acceleration at each of the three locomotion speeds revealed no statistically reliable differences between the speech and nonspeech conditions (Fig. 4). The mean magnitudes of maximum head acceleration for locomotion without speech were \pm 2.24 , \pm 5.43 , and $\pm 12.29 \mathrm{~m} / \mathrm{sec}^{2}$ for the 2,4 , and $6 \mathrm{mph}$ conditions, respectively. The mean magnitudes of maximum head acceleration for locomotion with speech were $\pm 1.90, \pm 5.22$, and \pm 10.79 $\mathrm{m} / \mathrm{sec}^{2}$. This suggests that differences in jaw orientation between speech and nonspeech conditions are not attributable to different magnitudes of head acceleration in the two conditions.

The magnitude of jaw movements during speech has been shown to be affected by both speaking rate and speech volume (Ostry et al., 1997). We performed two separate control studies to determine the extent to which these variables were correlated with head acceleration. The aim was to assess whether observed changes in jaw orientation might be attributable to these possible confounding factors. As a measure of speech rate, we used the mean duration of jaw movements associated with CVC production. The measure of speech volume was taken to be the mean amplitude of the rectified acoustic signal calculated over the voiced portion of the vowel. Analyses were performed separately for each subject. Pearson product-moment correlation coefficients were computed across all experimental conditions (three locomotion speeds $\times$ four CVC conditions) to measure the relationship between (1) speech rate and the magnitude of vertical head acceleration at the initial consonant, vowel, and final con-

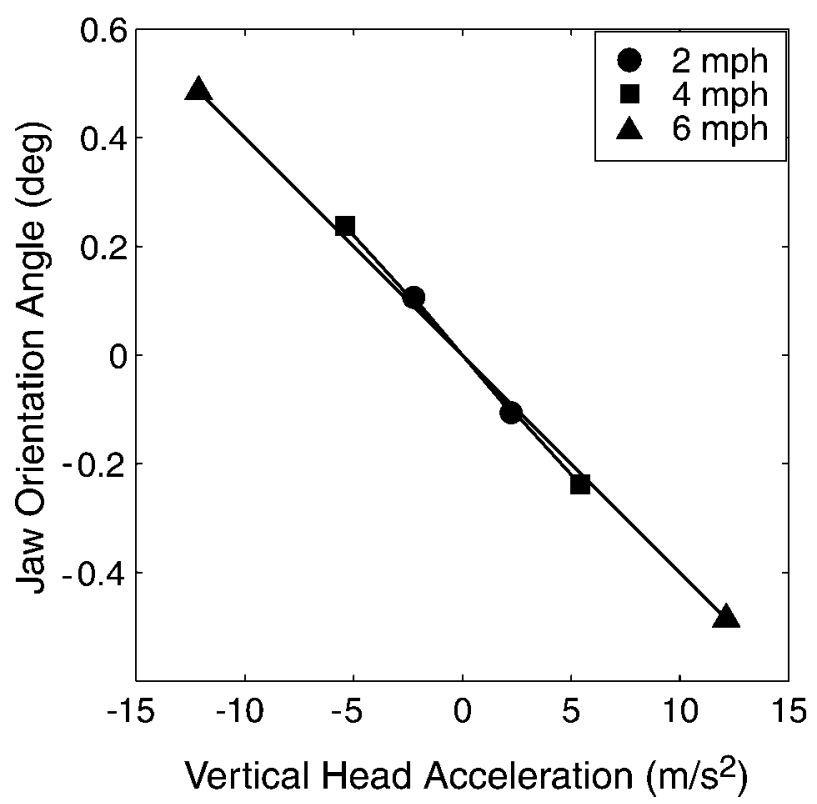

Figure 10. Simulation results for locomotion in the absence of speech. Control signals are not adjusted to take into account the effect of head acceleration on jaw orientation. Peak head acceleration is plotted on the $x$-axis (positive head accelerations are in the upward direction), and the corresponding jaw orientation is shown on the $y$-axis (the downward direction in the figure corresponds to jaw opening). The three lines show the simulated jaw displacement associated with three magnitudes of head acceleration (corresponding to the three locomotion speeds tested empirically). The direction and magnitude of the predicted effect at all three locomotion speeds are comparable with those observed empirically.

sonant and (2) speech volume and vertical head acceleration at the initial consonant, vowel, and final consonant. None of these relationships was found to be statistically reliable $(p>0.05)$.

\section{Simulations}

Two sets of simulations were performed in which the jaw model was used to predict the consequences of using control signals that are not adjusted to take into account the effect of vertical head acceleration on jaw orientation. The observed pattern of head acceleration associated with locomotion was approximated in the simulations by a sine wave, with the magnitude and frequency adjusted to match the empirical patterns reported above. The three magnitudes of maximum head acceleration were \pm 2.07 , \pm 5.33 , and $\pm 11.54 \mathrm{~m} / \mathrm{sec}^{2}$ for the 2,4 , and $6 \mathrm{mph}$ conditions, respectively. The three corresponding frequencies were 1.60, 2.10, and $2.66 \mathrm{~Hz}$. These values correspond to overall means across subjects, including speech and nonspeech conditions.

In the first set of simulations, the jaw equilibrium orientation was held constant at $3^{\circ}$ relative to occlusion, while the head accelerated upward and downward. This is analogous to the experimental condition in which subjects were walking or running on the treadmill without producing speech. The simulation results are shown in Figure 10. Peak vertical head acceleration is plotted on the $x$-axis, and the corresponding jaw orientation (relative to the specified orientation of $3^{\circ}$ ) is shown on the $y$-axis. The three sets of values show the simulated jaw displacement associated with the three magnitudes of head acceleration. In all cases, an upward head acceleration (positive) is associated with a lower position of the jaw, and a downward head acceleration (negative) is associated with a higher jaw position. The predicted magnitude of jaw displacement is shown to vary directly with the magnitude 


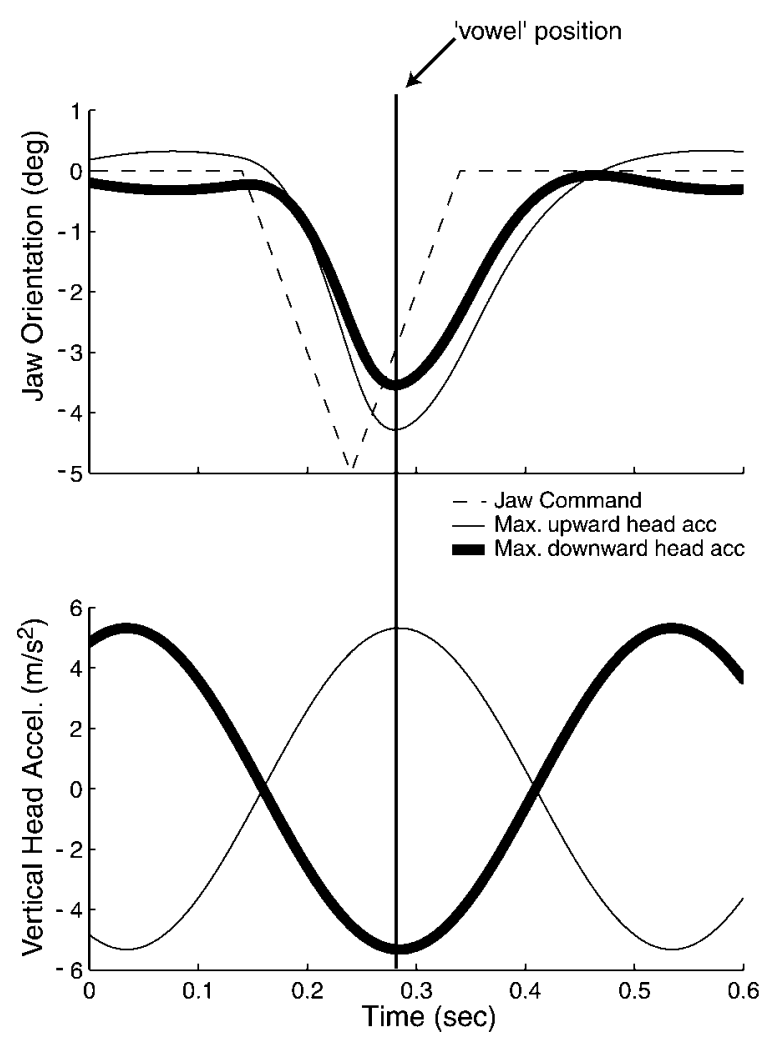

Figure 11. Sample of simulation results for locomotion with speech in the $4 \mathrm{mph}$ condition. Bottom, Simulated head acceleration. Top, The corresponding jaw orientation (solid lines), along with a single control signal to the jaw (dashed line). The two head acceleration records provide the maximum upward (shown as a thin line) and downward (shown as a thick line) acceleration at the "vowel" position of the jaw-opening and -closing movement. It can be seen that maximum upward head acceleration at the vowel position produces a lower jaw position, and maximum downward head acceleration produces a higher jaw position. acc or Accel., Acceleration; Max., maximum.

of head acceleration. Overall, the direction and magnitude of the predicted effect at all three locomotion speeds are comparable with those observed empirically.

A second set of simulations was used to generate predictions relating to the experimental condition of locomotion with speech. As in previous studies using the model (Laboissiere et al., 1996; Ostry et al., 1996; Shiller et al., 1999), we have used a control signal consisting of constant rate changes in the equilibrium orientation of the jaw coordinated with a constant rate shift in jaw horizontal equilibrium position (Fig. 11, top). This reproduces a naturalistic pattern of jaw motion in which movement amplitude is in the range of $4^{\circ}$ and $\mathrm{CVC}$ duration is $\sim 300 \mathrm{msec}$.

As in the first set of simulations described above, the simulated jaw movements were combined with three magnitudes of vertical head acceleration to model the effects of head movement on the jaw. The relative timing of head acceleration and jaw motion was varied to expose the jaw to a full range of upward and downward head accelerations. Specifically, for a given magnitude of head acceleration, simulations were repeated with the head acceleration signal phase shifted in increments of $5^{\circ}$. This allowed us to produce large upward and large downward head accelerations at each of the points of interest in the speech sequence (the initial and final consonants and the vowel).

Figure 12 shows an example of the procedure used to score the simulation results. In the figure, a subset of the simulated jaw

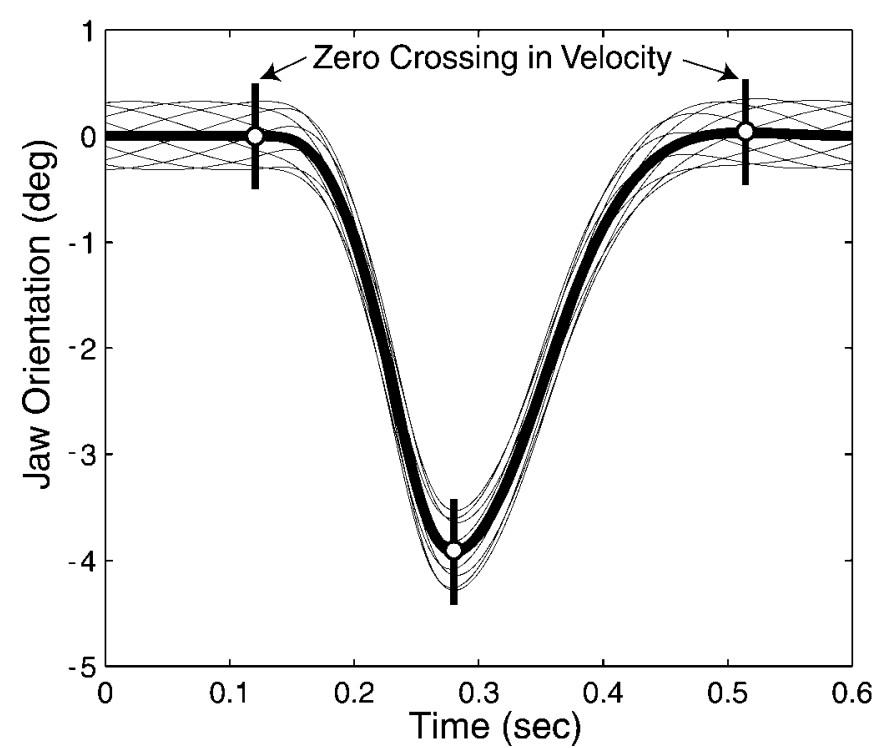

Figure 12. Example showing the procedure for scoring the simulated effects of head acceleration on jaw orientation. Three CVC positions (thick vertical lines) are identified on the basis of the jaw trajectory in the absence of head motion (thick line). A subset of simulated jaw movements during locomotion ( $4 \mathrm{mph}$ ) are shown with thin lines. The simulations are generated by shifting the timing of head acceleration relative to jaw movement.

movements is shown for the $4 \mathrm{mph}$ condition. Jaw motion in the absence of head movement is also shown as a thick line. Initial consonant, vowel, and final consonant positions are selected using this reference trajectory on the basis of zero-crossings in jaw velocity (indicated by thick vertical lines). At each of these three points, jaw orientations corresponding to maximum upward and maximum downward head acceleration are selected. This provides a prediction of the magnitude of the effect of head acceleration on jaw orientation. This entire procedure was repeated for each of the three magnitudes of maximum head acceleration associated with the three locomotion speeds.

Simulation results for the $4 \mathrm{mph}$ locomotion condition are shown in Figure 11. The bottom panel shows simulated vertical head acceleration, and the top panel shows the corresponding jaw orientation (solid lines), along with the specified control signal to the jaw (dashed line). The two head acceleration records in the bottom panel are those for which the maximum upward acceleration (thin line) and maximum downward acceleration (thick line) correspond in time to the vowel position of the jaw-opening and -closing movement. The resulting effect on jaw orientation is shown in the top panel. It can be seen that upward head acceleration is associated with a lower predicted jaw position at the vowel. Downward head acceleration is seen to result in a higher predicted jaw position.

The overall simulation results are presented in Figure 13. Only data for the vowel and the initial consonant are shown because the results for the two consonant positions were nearly identical. The simulations predict that in the absence of any change in control signals to the jaw, there should be an effect of head acceleration on jaw orientation that is similar in magnitude to that observed in locomotion without speech. When the head is accelerating in the downward direction, the jaw is displaced upward, and when the head accelerates in the upward direction, the jaw is displaced downward. The magnitude of the effect on 

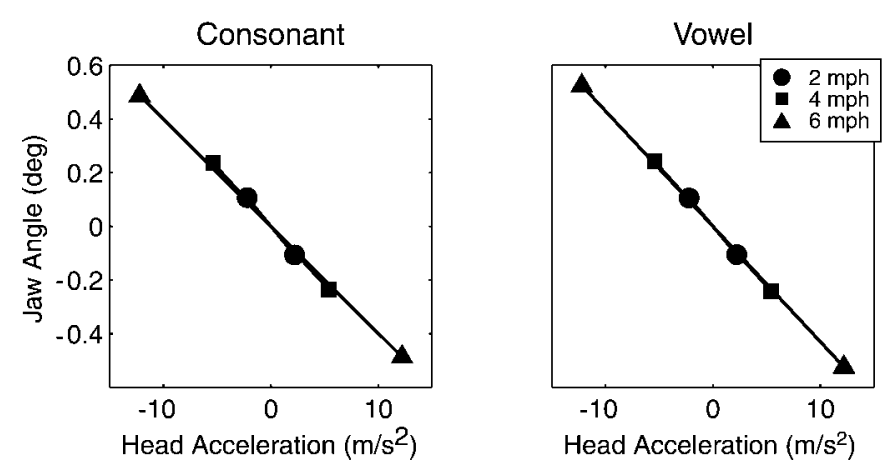

Figure 13. Simulation results for locomotion with speech shown for the initial consonant and vowel positions of the jaw-opening and -closing movement. The simulations predict that in the absence of compensation, an effect of head acceleration will be observed at both the consonant and vowel positions that is similar in magnitude to that observed in locomotion without speech.
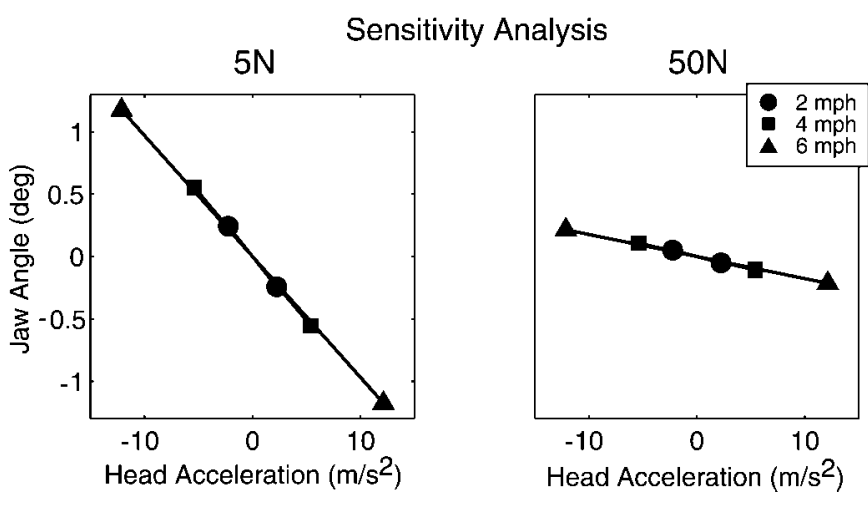

Figure 14. Results of the sensitivity analysis to test the influence of the cocontraction level (ranging from 5 to $50 \mathrm{~N}$ ) on the model predictions. The level of cocontraction is shown to influence the magnitude of the effect of head acceleration on jaw orientation, with higher cocontraction levels reducing the effect.

jaw orientation is shown to increase with the magnitude of head acceleration.

For all simulations presented here, a constant cocontraction level of $10 \mathrm{~N}$ (average modeled muscle force) was used. This value has been used previously in a number of simulation studies (Laboissiere et al., 1996; Ostry et al., 1996; Shiller et al., 1999) and was originally chosen by matching simulated jaw kinematics to a range of empirical data that are independent of the present study. In the present study, with a cocontraction value of $10 \mathrm{~N}$, we obtained simulation results that quantitatively match the empirical data. Specifically, the magnitude of changes to jaw orientation that arise because of head acceleration was similar in both simulated and empirical conditions of locomotion without speech.

A sensitivity analysis was performed to test the influence of changes in cocontraction level (ranging from 5 to $50 \mathrm{~N}$ ) on the model predictions described above. As expected, the level of cocontraction altered the magnitude of the effect of head acceleration on jaw orientation (Fig. 14). A cocontraction level of $5 \mathrm{~N}$ produced an effect on jaw orientation that was approximately double the magnitude of that observed empirically in locomotion without speech. A $50 \mathrm{~N}$ cocontraction level resulted in effects on jaw orientation that were approximately half as large. Nevertheless, at all cocontraction levels, an effect of head acceleration on jaw orientation was present. Furthermore, the magnitude of the effect on the jaw varied directly with the magnitude of head acceleration, and the direction of the effect remained unchanged; in all cases the jaw was displaced in a direction opposite that of vertical head acceleration.

\section{DISCUSSION}

In this study, we examined whether subjects adjust their control of jaw movements in speech to account for loads arising because of head acceleration during locomotion. When subjects walked or ran without speaking, jaw orientation was significantly affected by the direction and magnitude of head acceleration. Upward head acceleration produced a downward load on the jaw that resulted in lower jaw positions. Downward head acceleration had the opposite effect. The magnitude of the effect on jaw position varied directly with the magnitude of head acceleration. Thus under these conditions, compensation for acceleration-dependent loads was either absent or incomplete.

When locomotion was combined with speech, the effect of head acceleration on jaw orientation was examined at the initial consonant, vowel, and final consonant during the production of four different CVCs. In comparison with locomotion without speech, the effect of head acceleration on jaw position during production of the CVC was very small. Head acceleration significantly affected jaw motion only for the largest head accelerations tested (6 mph condition) and only during vowel production. However, even in this case, the size of the effect was smaller in magnitude than that observed during the slowest locomotion speed $(2 \mathrm{mph})$ in the nonspeech condition. Subjects thus appear to have been compensating for loads associated with head acceleration when producing jaw movements in speech.

We used a physiologically realistic model of the jaw to predict what would be observed in the absence of compensation. Specifically, we examined the predicted magnitude of the effect of head acceleration on jaw orientation when motor commands are not adjusted to take into account head acceleration-dependent loads. In the absence of compensation, simulations of locomotion with and without speech predicted the same effect of head acceleration on jaw orientation. In both cases, jaw orientation varied with the magnitude and direction of head acceleration, corresponding closely to the empirical data for locomotion without speech. The simulation results thus stand in contrast to the empirical results for locomotion with speech, supporting the idea that subjects compensate for self-generated loads acting on the jaw in speech.

An analysis was performed of the sensitivity of model predictions to the level of muscle cocontraction. The sensitivity analysis examined cocontraction levels ranging from 5 to $50 \mathrm{~N}$; a value of $10 \mathrm{~N}$ was used for predictions shown in Figures 10 and 13. The effect on the jaw of head acceleration was shown to vary in magnitude with the level of cocontraction such that higher levels of cocontraction were associated with a smaller size of the effect, and vice versa. This raises the interesting possibility that subjects may have used higher levels of cocontraction (and hence greater stiffness) to reduce the effect of head acceleration on the jaw during speech. Indeed, there is some empirical evidence that jaw muscle activity during speech is characterized by antagonist coactivation (Moore et al., 1988). Moreover, there is also evidence in studies of single-joint arm movement that increases in muscle coactivation can be used to offset the effects of load (Latash, 1992; Milner and Cloutier, 1993). In the model, however, a reduction of the size of the effect to a level comparable with that observed empirically during speech would require high levels of muscle cocontraction ( $\sim 50 \mathrm{~N}$ of average muscle force). 
It is also possible that subjects explicitly adjusted the timevarying control of the jaw to counteract the effect of head acceleration. Evidence of adjustment for movement-dependent loads has been reported previously in work on voluntary arm movement. In particular, predictive adjustments to EMG patterns that offset the effects of joint interaction torques have been observed in multijoint arm movement (Sainburg et al., 1993; Almeida et al., 1995; Cooke and Virji-Babul, 1995; Sainburg et al., 1995; Gribble and Ostry, 1999; Koshland et al., 2000).

Motion-dependent loads to the jaw have been studied previously in locomotion (Murray, 1967; Lund et al., 1984). In both cats and humans, the intrinsic stiffness of jaw muscles and stretch reflexes have been shown to counteract loads associated with head motion (Lund et al., 1984). Other reflex-based compensations may arise in vestibular receptors and neck muscles that influence jaw-closer motoneurons (Funakoshi and Amano, 1973; Griffiths et al., 1983). In the present empirical study, compensation was not observed when locomotion alone was tested; jaw position varied directly with the magnitude and direction of head acceleration. This indicates that in the absence of overall changes to reflex excitability in the context of speech, the effect of reflexes and muscle mechanical properties are insufficient to compensate fully for the effect of head motion on the jaw. The simulations are consistent with this conclusion, because the model, which includes passive muscle stiffness and active muscle mechanical properties as well as activation because of the stretch reflex, predicts an effect of head acceleration on jaw position even at the slowest locomotion speed. It remains unknown whether reflex excitability of jaw muscles is comparable in speech and nonspeech conditions.

Head movement during locomotion has been examined in a number of previous studies, many of which have demonstrated that during the step cycle, the head not only translates vertically through space (to a degree that depends on locomotion speed) but also rotates in the sagittal plane (Bloomberg et al., 1992; Crane and Demer, 1997; Hirasaki et al., 1999). An important feature of these studies is that subjects were instructed to fixate a visual target while walking or running. The head rotation thus appears compensatory in nature; the effect of upward or downward head translation on visual fixation is offset by a sagittal plane rotation of the head in the opposite direction. In the present study, as in naturally occurring locomotion, subjects were not instructed to fixate visually. As a result, head orientation in the present study showed no systematic relationship to the timing and magnitude of vertical head acceleration.

Motorized treadmills are routinely used to study a wide range of physiological variables associated with locomotion. Nevertheless, it is reasonable to suspect that there may be differences between treadmill and overground locomotion. In previous studies, it has been found that compared with overground locomotion, treadmill locomotion involves a shorter stride length and increased stride frequency (by $\sim 7-10 \%$ ) (Elliott and Blanksby, 1976; Murray et al., 1985; Stolze et al., 1997; Wank et al., 1998). Such differences indicate that treadmill locomotion might involve a smaller amplitude of vertical head acceleration compared with overground locomotion. Nevertheless, in the present study, the magnitude of head acceleration was sufficient to affect jaw orientation during locomotion without speech. Furthermore, the magnitude of the effect on jaw orientation was apparently sufficient to provoke subjects to compensate during speech production.

We have demonstrated by use of the model that changes to the cocontraction level might contribute in some part to the observed compensation for the effects of head motion on the jaw during speech. More generally, the compensation might arise via a combination of changes to the cocontraction level and accompanying anticipatory changes to the time-varying motor command underlying jaw movement. In addition, changes in the excitability of jaw muscle motoneurons to feedback from sensors may play a role. The specific balance of these sources of compensation may be explored via a combination of empirical studies involving recordings of jaw muscle activity in conjunction with modeling studies in which changes in the form of the control signal are inferred by matching model predictions to corresponding empirical patterns of jaw motion (see Gribble and Ostry, 2001).

In the condition in which speech was combined with locomotion, a potential issue arises related to the data selection procedure. The analyses in the present study are based on the selection of specific points within the jaw kinematic record corresponding to consonant and vowel production, identified on the basis of zero-crossings in jaw velocity. Head acceleration potentially affects the kinematic pattern of the jaw and hence might influence the position and timing of these points. Specifically, the presence of head acceleration might lead to situations in which the jaw does not reach zero velocity during consonant production immediately before or after the large-amplitude movement associated with vowel production. In the present study this occurred infrequently (2-3\% of cases), and in those cases the data were excluded from analysis. For the most part, jaw movements in the presence of locomotion had the same general form as did those in the absence of locomotion, and hence the use of zero-crossings in velocity as a selection criterion introduced few problems.

The generality of the compensation observed here to jaw movement in speech as opposed to other articulators and other orofacial behaviors remains unknown. In future work, it would be worthwhile to investigate whether compensation for head acceleration during locomotion extends to other articulators such as the tongue or the lips. Additionally, it may be the case that compensation is not specific to speech but can be found in other goal-directed behaviors such as mastication.

\section{REFERENCES}

Abbink JH, van der Bilt A, Bosman F, van der Glas HW (1998) A comparison of jaw-opener and jaw-closer muscle activity in humans to overcome an external force counteracting jaw movement. Exp Brain Res 118:269-278.

Abbink JH, van der Bilt A, Bosman F, van der Glas HW, Erkelens CJ, Klaassen MFH (1999) Comparison of external load compensation during rhythmic arm movements and rhythmic jaw movements in humans. J Neurophysiol 82:1209-1217.

Almeida GL, Hong DA, Corcos D, Gottlieb GL (1995) Organizing principles for voluntary movement: extending single-joint rules. J Neurophysiol 74:1374-1381.

Bloomberg JJ, Reschke MF, Huebner WP, Peters BT (1992) The effects of target distance on eye and head movement during locomotion. Ann NY Acad Sci 656:699-707.

Conditt MA, Gandolfo F, Mussa-Ivaldi FA (1997) The motor system does not learn the dynamics of the arm by rote memorization of past experience. J Neurophysiol 78:554-560.

Cooke JD, Virji-Babul N (1995) Reprogramming of muscle activation patterns at the wrist in compensation for elbow reaction torques during planar two-joint arm movements. Exp Brain Res 106:177-180.

Crane BT, Demer JL (1997) Human gaze stabilization during natural activities: translation, rotation, magnification, and target distance effects. J Neurophysiol 78:2129-2144.

De Wolf SD, Slijper H, Latash ML (1998) Anticipatory postural adjustments during self-paced and reaction time movements. Exp Brain Res 121:7-19.

Elliott BC, Blanksby BA (1976) A cinematographic analysis of overground and treadmill running by males and females. Med Sci Sports 8:84-87.

Feldman AG (1986) Once more on the equilibrium-point hypothesis (lambda model) for motor control. J Mot Behav 18:17-54.

Feldman AG, Adamovich SV, Ostry DJ, Flanagan JR (1990) The origin of electromyograms: explanations based on the equilibrium point hy- 
pothesis. In: Multiple muscle systems: biomechanics and movement organization (Winters J, Woo S, eds), pp 195-213. New York: Springer. Flanagan JR, Wing A (1997) The role of internal models in motion planning and control: evidence from grip force adjustments during movements of handheld loads. J Neurosci 17:1519-1528.

Flanagan JR, Ostry DJ, Feldman AG (1993) Control of trajectory modifications in target-directed reaching. J Mot Behav 25:140-152.

Funakoshi M, Amano N (1973) Effect of the tonic neck reflex on the jaw muscles of the rat. J Dent Res 52:668-673.

Gandolfo F, Mussa-Ivaldi FA, Bizzi E (1996) Motor learning by field approximation. Proc Natl Acad Sci USA 93:3843-3846.

Goodbody SJ, Wolpert DM (1998) Temporal and amplitude generalization in motor learning. J Neurophysiol 79:1825-1838.

Gribble PL, Ostry DJ (1998) Independent coactivation of shoulder and elbow muscles. Exp Brain Res 123:355-360.

Gribble PL, Ostry DJ (1999) Compensation for interaction torques during single and multi-joint limb movements. J Neurophysiol $82: 2310-2326$

Gribble PL, Ostry DJ (2000) Compensation for loads during arm movements using equilibrium-point control. Exp Brain Res 135:474-482.

Griffiths C, Thexton AJ, McGarrick JD (1983) The effect of head position upon jaw reflexes in the cat. Arch Oral Biol 28:359-391.

Guenther FH (1995) Speech sound acquisition, coarticulation, and rate effects in a neural network model of speech production. Psychol Rev 102:594-621.

Guenther FH, Hampson M, Johnson D (1998) A theoretical investigation of reference frames for the planning of speech movements. Psychol Rev 105:611-633.

Hirasaki E, Moore ST, Raphan T, Cohen B (1999) Effects of walking velocity on vertical head and body movements during locomotion. Exp Brain Res 127:117-130.

Honda M, Kaburagi T (2000) Speech compensation to dynamical structural perturbation of the palate shape. Proceedings of the 5 th Seminar on Speech Production, Kloster Seeon, Bavaria, pp 21-24.

Horak FB, Esselman P, Anderson ME, Lynch MK (1984) The effects of movement velocity mass displaced and task certainty on associated postural adjustments made by normal and hemiplegic individuals. J Neurol Neurosurg Psychiatry 47:1020-1028.

Houde JF, Jordan MI (1998) Sensorimotor adaptation in speech production. Science 279:1213-1216.

Humphrey DR, Reed DJ (1983) Separate cortical systems for control of joint movement and joint stiffness: reciprocal activation and coactivation of antagonist muscles. In: Motor control mechanisms in health and disease (Desmedt J, ed), pp 347-372. New York: Raven.

Koshland GF, Galloway JC, Nevoret-Bell CJ (2000) Control of the wrist in three-joint arm movements to multiple directions in the horizontal plane. J Neurophysiol 83:3188-3195.

Laboissiere R, Ostry DJ, Feldman AG (1996) Control of multi-muscle systems: human jaw and hyoid movements. Biol Cybern 74:373-384.

Lackner JR, DiZio P (1994) Rapid adaptation to coriolis force perturbations of arm trajectory. J Neurophysiol 72:299-313.

Latash ML (1992) Independent control of joint stiffness in the framework of the equilibrium-point hypothesis. Biol Cybern 67:377-384.

Lund JP, Drew T, Rossignol S (1984) A study of jaw reflexes of the awake cat during mastication and locomotion. Brain Behav Evol 25:146-156.

McDevitt WE (1989) Functional anatomy of the masticatory system. London: Wright.

McFarland DH, Baum SR (1995) Incomplete compensation to articulatory perturbation. J Acoust Soc Am 97:1865-1873.

McFarland DH, Baum SR, Chabot V (1996) Speech compensation to structural modifications of the oral cavity. J Acoust Soc Am 100:1093.

Milner TE, Cloutier C (1993) Compensation for mechanically unstable loading in voluntary wrist movement. Exp Brain Res 94:522-532.

Moore CA, Smith A, Ringel RL (1988) Task-specific organization of human jaw muscles. J Speech Hear Res 31:670-680.

Murray MP (1967) Gait as a total pattern of movement. Am J Phys Med 46:290-333.

Murray MP, Spurr GB, Sepic SB, Gardner GM, Mollinger LA (1985) Treadmill vs. floor walking: kinematics, electromyogram, and heart rate. J Appl Physiol 59:87-91.

Ostry DJ, Munhall KG (1994) Control of jaw orientation and position in mastication and speech. J Neurophysiol 71:1528-1545.

Ostry DJ, Gribble PL, Gracco VL (1996) Coarticulation of jaw movements in speech production: is context-sensitivity in speech kinematics centrally planned? J Neurosci 16:1570-1579.

Ostry DJ, Vatikiotis-Bateson E, Gribble PL (1997) An examination of the degrees of freedom of human jaw motion in speech. J Speech Lang Hear Res 40:1341-1351.

Perkell JS, Matthies ML, Lane H, Guenther FH, Wilhelms-Tricarico R, Wozniak J, Guiod P (1997) Speech motor control: acoustic goals, saturation effects, auditory feedback and internal models. Speech Communication 22:227-250.

Sainburg RL, Poizner H, Ghez C (1993) Loss of proprioception produces deficits in interjoint coordination. J Neurophysiol 70:2136-2147.

Sainburg RL, Ghilardi MF, Poizner H, Ghez C (1995) Control of limb dynamics in normal subjects and patients without proprioception. J Neurophysiol 73:820-835.

Scheideman GB, Bell WH, Legan HL, Finn RA, Reich JS (1980) Cephalometric analysis of dentofacial normals. Am J Orthod 78:404-420.

Shadmehr R, Mussa-Ivaldi F (1994) Adaptive representation of dynamics during learning of a motor task. J Neurosci 14:3208-3224.

Shiller DM, Gribble PL, Ostry DJ (1999) Effects of gravitational load on jaw movement in speech. J Neurosci 19:9073-9080.

Stolze H, Kuhtz-Buschbeck JP, Mondwurf C, Boczek-Funcke A, John K, Deuschl G, Illert M (1997) Gait analysis during treadmill and overground locomotion in children and adults. Electroencephalogr Clin Neurophysiol 105:490-497.

Tiede MK, Masaki S, Vatikiotis-Bateson E (2000) Contrasts in speech articulation observed in sitting and supine conditions. Proceedings of the 5th Seminar on Speech Production, Kloster Seeon, Bavaria, pp 25-28.

Wank V, Frick U, Schmidtbleicher D (1998) Kinematics and electromyography of lower limb muscles in overground and treadmill running. Int J Sports Med 19:455-461.

Zajac F (1989) Muscle and tendon: properties, models, scaling, and application to biomechanics and motor control. CRC Crit Rev Bioeng 17:359-415. 